\title{
Análisis Multivariado de Parámetros Fisicoquímicos del Relleno Sanitario de São Giacomo de Caxias do Sul, RS en la de Degradaciòn de Polipropilene
}

\author{
Ana M. C. Grisa, Mara Zeni \\ Centro de Ciências Exatas e Tecnologia, UCS \\ Aldemar Muñoz \\ Universidad del Tolima, Colombi
}

\begin{abstract}
Resumen: El presente artículo describe la aplicación de herramientas de software al estudio de degradación/biodegradación de polímeros commodity polipropileno (PP) en el relleno sanitario de São Giacomo de Caxias do Sul, RS. Se hace inicialmente una descripción del relleno sanitario, luego se describen las variables fisicoquímicas que intervienen en el proceso, se organizan las matrices de datos y se aplica el algoritmo PCA para interpretar las variables correlacionadas con la degradación y la actividad biológica. En el análisis de los datos multivariados se usaron las herramientas Matlab, InfoStat, con el fin de establecer los parámetros fisicoquímicos involucrados en la degradación/biodegradación, y determinar los componentes correlacionados con el fin de poder predecir el comportamiento de los parámetros y la degradación del polímero en el relleno sanitario. El análisis de componentes principales (PCA) permitió la reducción de 12 variables del 2 componentes principales que muestram a correlación de las variables. Los parámetros importantes son: relación DBO/DQO que miden la biodegradabilidad y nitrógeno amoniacal un indicativo de actividad biológica. Los resultados del análisis PCA describen la estructura y las interrelaciones de variables originales en el fenómeno de biodegradación de la materia orgánica a través del análisis e interpretación de los componentes principales.
\end{abstract}

Palabras-clave: Relleno sanitario, análisis PCA, degradación, componentes principales, polipropileno.

\section{Multivariate Analysis of Physicochemical Parameters of the Landfill São Giácomo in Caxias do Sul, RS in the Degradation of Polypropylene}

\begin{abstract}
This article describes the application of software tools to investigate the degradation /biodegradation of the commodity polymer polypropylene (PP) in the sanitary filling of Sao Giacomo in Caxias Do Sul, RS. A description is given of the sanitary filling, in addition to the physicochemical variables that are relevant for the process. The data are organized in matrices and a Principal Component Analysis (PCA) algorithm is applied to interpret the variables correlated with the degradation and biological activity. For analyzing the data, use was made of the tools Matlab and InfoStat, with the purpose of establishing the physicochemical parameters involved in the degradation/biodegradation, and for determining the correlated components in order to predict the behavior of the parameters and the polymer degradation in the sanitary filling. The PCA analysis allowed one to describe the structure and interrelations of the original variables for the biodegradation of organic matter. The important parameters were found to be DQO and DBO which measure biodegradability and ammoniac nitrogen, an indicative of biological activity.
\end{abstract}

Keywords: Landfill, multivariate, degradation, principal components, polypropylene.

\section{Introduccion}

Un relleno sanitario es un ecosistema complejo, donde a través de interacciones bioquímicas de diferentes especies microbianas ocurren procesos físicos, biológicos, con una descomposición de materia orgánica y una conversión de materiales en monómeros y olígomeros ${ }^{[1]}$.

La generación diaria de Caxias do Sul es del orden de 275 t de residuos la fracción de material polimérico en el residuo sólido domiciliario corresponde a un $14,6 \%$ del total $^{[2]}$, siendo $5 \%$ de polipropileno (PP).
La disposición de residuos en células de relleno fue implantada según las normas brasileras para relleno sanitario (NBR 8418/1984 e NBR 8419/1984), y el sistema de remediación de São Giácomo com licencia de operación de órgano ambiental estadual FEPAM (Fundação Estadual de Proteção Ambiental) y es monitoreado mensualmente.

La disposición de los residuos sólidos, en celula C4, relleno São Giácomo de forma controlado permitió condiciones adecuadas al desarrollo del proceso de digestion anaerobia de la fracción orgánica en los residuos

Autor para correspondência: Mara Zeni, Centro de Ciências Exatas e Tecnologia, Universidade de Caxias do Sul, Rua Francisco Getulio Vargas, 1130, Bloco G, Petrópolis, Caixa Postal 1352, CEP: 95070-970, Caxias do Sul, RS, Brasil. E-mail: mzandrad@ucs.br 
y el desarrollo de la actividad microbiana en el interior de las células de relleno, asociado a factores ambientales, promueven la percolación de cantidades de lixiviado y su posterior tratramiento, según APHA, AWWA \& WPCF (1989) ${ }^{[3]}$.

La degradación de los residuos de polímeros en el relleno sanitario se puede producir mediante la oxidación térmica, foto-degradación y/o la acción de microbios. ${ }^{[4]}$ Los microorganismos en el relleno sanitario, durante su metabolismo, liberan enzimas que son responsables de la transformación de una sustancia en otra. Las células microbianas disponen de un arsenal que son capaces de actuar sobre los productos químicos sintéticos derivados de las actividades antropogénicas ${ }^{[5]}$.

Los rellenos viejos tienen una cantidad significativa de material biologicamente inerte, lo que es indicado por la baja relación $\mathrm{DBO} / \mathrm{DQO}$ o por la gran diferencia entre estos valores. La relación $\mathrm{DBO} / \mathrm{DQO}$, indica el porcentaje de materia orgánica que es biodegradable y que disminuye a medida que el relleno evoluciona. Inicialmente esta relación es de 0,5 a 0,8, pasando a 0,07, 0,08 después de varios años. Otros autores describen bandas como: Robinson $(1979)^{[6]}$, una relación DBO/DQO asume valores superiores a 0,4 durante la fase ácida e inferiores e iguales a 0,4 durante la fase metanogénica.

Una deterioración/degradación del material polimérico es una función de su estructura, presencia de población microbiana y condiciones ambientales para el desarrollo del crecimiento microbiano ${ }^{[1,3]}$.

Grisa y Zeni ${ }^{[7]}$ estudiaron la degradación/biodegradación de polímeros commoditie polipropileno (PP), en condiciones bióticas/abióticas en el relleno sanitario de São Giácomo de Caxias, Brasil. El PP C4 - 18 m posee energía de activación diferente de las demás muestras que puede ser debido a la tacticidad del PP y la ruptura randómica. Los análisis de IV(FT-IR) demuestran que hay evidencias de un mecanismo termooxidativo y cambios significativos de cristalinidad.

En el relleno sanitário las regiones amorfas del PP inicialmente son las más sencibles a la degradación oxidativa y las impurezas presentes actuan como iniciadores de fotodegradación ${ }^{[8]}$.

En este estudio un conjunto de variables fisicoquímicas medidas a diferentes tiempos de vida de la celda 4 constituyen un modelo multivariado. El análisis y, el uso de métodos multivariados a través de software, se destina al establecimiento de parámetros fisicoquímicos involucrados en la degradación/ biodegradación, en la exploración de los componentes que poseen correlación, la predicción del comportamiento de los parámetros fisicoquímicos y la degradación del polímero en el relleno.

El análisis de componentes principales (PCA) es un método estadístico multivariado de simplificación o reducción de la dimensión de una tabla de casos o variables com datos cuantitativos, para obtener una tabla de menor número de variables, combinación lineal de las iniciales que se denominan componentes (CPs). La PCA reduce el número de variables en un conjunto de datos, conteniendo toda la información original, agrupando aquellas que fornecen informaciones semejantes y que están altamente correlacionadas. Su aplicación es directa sobre cualquier conjunto de variables, permite describir la estructura y las interrelaciones de las variables originales en el fenómenos en estudio a partir de los componenetes obtenidos. La reducción de muchas variables a pocos componentes puede simplicar la ampliación sobre otras técnicas multivariadas ${ }^{[9]}$.

En el estudio de la aplicación de herramientas matemáticas y de herramientas de software al relleno sanitario en cuestión se tiene un conjunto de variables fisicoquímicas medidas sobre diferentes muestras pertenecientes a celda $\mathrm{C} 4$ del relleno. El análisis que se propone esta basado en el estudio de la degradación/biodegradación que se presenta en la celda en el relleno sanitario utilizando analisis de dados multivariados con las herramientas Matlab, infoStat y Norm. PCA es uno de los diversos métodos multivariados que permite explorar patrones en los datos, como los componentes se comportan en forma similar, las celdas que pertenecen a un grupo similar, y en general como se puede predecir el comportamiento a partir de información disponible.

El principal uso delPCA estarelacionadocon la explicación de la estructura de varianzas y covarianzas de una serie de variables originales, mediante unas pocas combinaciones lineales de ellas, buscando generar nuevas variables que puedan expresar la información contenida en el conjunto original de datos y reducir la dimensionalidad del problema estudiado. Las nuevas variables generadas se denominan componentes principales. El análisis es esencialmente descriptivo y tiene una interpretación geométrica ${ }^{[10-11]}$. Lo que se persigue es extraer información de una matriz multivariada conformada por variables y muestras tomadas en el tiempo. De igual manera en la aplicación del relleno sanitario se tienen las variables fisicoquímicas y muestras tomada en el tiempo a partir de lixiviados recolectados in situ. La idea entonces es reducir el número de variables a un número que de explicación a la máxima varianza de los datos, pues se tienen muchas observaciones (muestras) $\mathrm{y}$ un alto numero de dimensiones. Esta herramienta permite razonar sobre la información con más profundidad y por esta razón es necesario reducir los datos a un conjunto de factores mas pequeños, los cuales son combinaciones de variables observadas y pueden ser bases más efectivas para profundizar, y corresponden a los componentes principales. Aun si el significado físico de lo que se interpreta es oscuro, los datos observados son descritos en términos de estos factores en lugar de describirlos en términos de las variablesdimensiones originales.

El escogitamiento de los componentes principales que son utilizados en la descripción de los datos es realizada, tomando en consideración el porcentaje de varianza descritas por las CPs y la varianza residual ${ }^{[12]}$.

Las nuevas variables generadas se denominan componentes principales, el cual es un análisis esencialmente descriptivo y 
tiene una interpretación geométrica e permitem a construção de um modelo de regressão linear ${ }^{[13]}$.

Mediante el uso de PCA, se describe la estructura e interrelación de variables que originan el fenómeno de biodegradación de la materia orgánica, obtenidas agrupando variables que tienen información semejante (altamente correlacionadas).

PCA es una técnica de reconocimiento de patrones utilizados para analizar la estructura de datos multivariante, la reducción de la dimensionalidad de la matriz original de datos de la variabilidad máxima. Proporciona un nuevo conjunto de variables (componentes principales) que facilitan el descubrimiento de patrones ocultos en el conjunto de datos $^{[11,14,15]}$.

Les componentes principales son combinaciones lineales de las medidas originales y para visualizar la naturaleza de los datos multivariantes en un espacio pequeño, a menudo en dos plano tridimensional. Estos son diseñados de tal modo que se vea como información estadística mucho más, es decir, el primer factor (CP1) tiene una variación máxima de los datos en una dirección. En otras palabras, esta es la dirección que describe la mayor difusión de las muestras. El segundo factor (CP2) se describe la varianza máxima restantes y así sucesivamente. El grafico scores y loadings son muy útiles para este punto de vista ${ }^{[16]}$.

\section{Materiales y Metodos}

El polipropileno (PP) fue colectado a través de perforaciones en la celda $\mathrm{C} 4 \mathrm{y}$ otras celdas del relleno municipal y con diferentes profundidades ( 8 a $18 \mathrm{~m}$ ). Después el material fue lavado según normas ASTM D 6288-98 y fueron realizados análisis termo gravimétricos, estructurales y morfológicos para la determinación de de la degradación del material.

En el análisis termogravimétrico, se identificaron modificaciones termogravimétricas (TG y DTG) en las películas poliméricas que fueron analizadas usando el método de Freeman-Carrol. En este método, la energía de activación (Ea) de las muestras fue menor que de la (Ea) del polímero virgen y la (Ea) crece con el tiempo de depositación y las profundidades de las celdas del relleno.

Con el fin de aplicar PCA. Es necesario un pre-tratamiento de los datos para eliminar "outliers" y valores faltantes ("missing values"). El proceso inicia con la generación de la matriz de covarianza del conjunto de datos. En este trabajo las columnas representas las variables fisicoquímicas y las filas las muestras en el tiempo.

En este trabajo las columnas representan las variables fisicoquímicas y las filas las muestras en el tiempo. Se utiliza Matlab para escalar los datos (MPCA: Multi-way Principal Component Analysis) y Norm con el fin de completar los datos faltantes en cada una de las matrices de cada celda. El análisis de PCA se realizó haciendo uso del paquete de software Infostat.
El trabajo corresponde al estudio en condiciones bióticas del relleno sanitario de Sao Giacomo de Caxias Do Sul, RS, Brasil utilizando el método exploratorio de análisis multivariado de parámetros fisicoquímicos del lixiviado $\mathrm{pH}$, alcalinidad, sólidos volátiles totales (STV), demanda química de oxigeno, (DQO), demanda bioquímica de oxigênio(DQO), nitrogênio Total (N.T.), nitrogênio amoniacal ( $\left.\mathrm{N}-\mathrm{NH}_{3}\right)$, cadmio $(\mathrm{Cd})$, cobre $(\mathrm{Cu})$, ferro $(\mathrm{Fe})$, manganês $(\mathrm{Mn})$ e zinco $(\mathrm{Zn})$, producido en la celda $\mathrm{C} 4$ del relleno sanitario a lo largo del 5 anos, apuntando a la determinación de procesos de degradación/ biodegradación. En Grisa (2004) ${ }^{[17]}$ se presenta una primera discusión sobre análisis de PCA en este relleno sanitario.

\section{Resultados y Conclusiones}

El PP es un polímero altamente susceptibles a la fotodegradación cuando son expuestos a la intemperie ${ }^{[18]}$. La energía absorbida por la influencia del calor, luz, etc estrés mecánico, la rotura causa de enlaces $\mathrm{CH}$ de los servicios de carbono, que tienen vínculos más débiles que los otros carbones, lo que genera radicales libres que son capaces de reaccionar con el oxígeno para formar peróxidos e hidroperóxidos, que a su vez producen más radicales libres resultantes de la división de cadenas $^{[19-22]}$. El PP puede presentar varios grupos cromóforos (grupos generadores de radicales libres) como iniciadores de la degradación del PP que inducen a la ruptura de la cadena.

Según Suat et al. (2008) ${ }^{[23]}$, la degradación térmica de los residuos PP muestran las diferentes etapas de la pérdida de masa, que se producen a temperaturas entre 310 y $475^{\circ} \mathrm{C}$.

Em la aplicación del método de Freeman-Carroll dos energías de activación a los picos de degradación para el PP colectado del relleno, la energia de activación disminuye con el tiempo de rellenamiento y posee altos coeficientes de correlación.

Los valores de energía de activación (EA) para el PP por la aplicación del método de Freeman-Carroll, teniendo en cuenta la región de la degradación y la velocidad de calentamiento a $10{ }^{\circ} \mathrm{C} / \mathrm{min}$ y el coeficiente de correlación de la DTG se muestran Tabla 1.

Los valores de Ea obtenidos a partir de la curva de TG a una temperatura de degradación están cerca de la literatura, los valores de segundos y Chan \& Balke ${ }^{[24]}$, 279-256 J.mol ${ }^{-1}$.

En la aplicación del metdodo Freeman-Carroll se obtuvieron dos energías de activación relativas del pico de la degradación a $460,41^{\circ} \mathrm{C}$ para las muestras de PP virgen y C4 muestra PP-18m, muestra tomada en el relleno sanitario con una mayor tiempo de permanencia se obtuvieron sólo una energía de activación.

El PP siendo una polioefina su degradación depende de su estructura, de la interacción entre las cadenas, de la rigidez, de las ramificaciones de tacticidad y de la cristalinidad, siendo que las regiones amorfas son verdaderamente sensibles la degradación oxidativa. Los hidroperóxidos y carbonilos, 
Tabla 1. Análisis termogravimétrico del $\mathrm{PP}\left(10^{\circ} \mathrm{C} / \mathrm{min}\right.$ em $\left.\mathrm{N}_{2}\right)$.

\begin{tabular}{lcccc}
\hline Polímero & $\begin{array}{c}\text { Temperatura } \\
\left({ }^{\circ} \mathbf{C}\right)\end{array}$ & $\begin{array}{c}\text { \% } \\
\text { perdida } \\
\text { de peso }\end{array}$ & $\begin{array}{c}\text { Ea } \\
\left(\mathbf{k J \cdot m o l}{ }^{-1}\right)\end{array}$ & $\mathbf{r}$ \\
\hline PP virgen & 460,41 & 98,17 & 133,91 & 0,9979 \\
& & & 280,49 & 0,9986 \\
PP - C4 -18 m & 465,35 & 97,68 & 299,21 & 0,9997 \\
\hline
\end{tabular}

pigmentos, cargas lubricantes o plastificantes actuan como iniciadores de la fotodegradación ${ }^{[25]}$.

El PP C4 - $18 \mathrm{~m}$ posee energía de activación diferente de las demás muestras que puede ser debido a la tacticidad del PP y la ruptura randómica ${ }^{[26]}$.

Através del estudio de la microscopía electrónica de los materiales es posible visualizar diferencias em la superficie de la muestra de PP las cuales presentan escamas y fisuras ${ }^{[17,27]}$.

La motivación para aplicar PCA es encontrar una nueva variable o unas pocas variables nuevas que representen tanto como sea posible la varianza en el conjunto de datos.

Los autovectores constituyen un conjunto de vectores bases para graficar los datos y los autovalores corresponden a la variabilidad de los datos en cada una de las direcciones dadas por los autovectores. Son medidas de variabilidad mientras que los autovectores expresan la dirección de la variabilidad.

Los cuatro primeros componentes principales estandarizados (el paquete de software InfoStat permite estandarizar los datos), con eigenvalues mayores de uno, explican el $81 \%$ de la estructura de varianzas y covarianzas de los datos se pueden ver en Tabla 2.

Se asumieron los dos primeros componentes principales como satisfactorios al explicar un $66 \%$ de la variación muestral (estructura de varianzas y covarianzas de los datos), lo que equivale pasar de 12 variables a 2 componentes principales. Un $66 \%$ se considera suficiente y el espacio bidimensional es de más fácil comprensión para el análisis de resultados.

Las expresiones matemáticas que muestran el comportamiento de las variables dos CPs de la célula C4 se presentan nas Ecuaciones 1 e 2 .

$\mathrm{CP} 1=0,37 \mathrm{DBO}+0,35 \mathrm{DQO}+0,34 \frac{\mathrm{DBO}}{\mathrm{DQO}}+0,33 \mathrm{Zn}+0,30 \mathrm{Cd}+$

$+0,30 \mathrm{Cu}+0,26 \mathrm{STV}+0,02 \mathrm{Fe}$

$\mathrm{CP} 2=0,58 \mathrm{Alcal}+0,38 \mathrm{NT}+0,25 \mathrm{~N}-\mathrm{NH}_{3}+0,19 \mathrm{Cu}+0,18 \mathrm{DBO}+$

$+0,18 \mathrm{DQO}+0,15 \mathrm{pH}+0,12 \mathrm{STV}+0,15 \mathrm{Fe}+0,05 \mathrm{Zn}+0,04 \frac{\mathrm{DBO}}{\mathrm{DQO}}$

La importancia de las variables se puede inferir por el valor de sus coeficientes y la distancia desde el origen. Se observa en general sin embargo, alto predominio de las variables DBO y DQO a lo largo del primer componente principal $\mathrm{CP} 1$, mientras un buen predominio de las variables $\mathrm{NT}, \mathrm{N}-\mathrm{NH}_{3}$ y alcalinidad, al igual que de los metales a lo largo del componente CP2. El primer componente CP1 explica lo relacionado con la degradación anaeróbica de los microorganismos sobre la materia orgánica contenida en la
Tabla 2. Autovectores.

\begin{tabular}{lrc}
\hline Variables & e1 & e2 \\
\hline $\mathrm{pH}$ & $-0,28$ & 0,15 \\
Alcal & $-0,08$ & 0,58 \\
$\mathrm{STV}$ & 0,26 & 0,12 \\
$\mathrm{DQO}$ & 0,35 & 0,18 \\
$\mathrm{DBO}$ & 0,37 & 0,18 \\
$\mathrm{NT}$ & $-0,26$ & 0,38 \\
$\mathrm{~N}-\mathrm{NH} 3$ & $-0,34$ & 0,25 \\
$\mathrm{Cd}$ & 0,30 & 0,09 \\
$\mathrm{Cu}$ & 0,30 & 0,19 \\
$\mathrm{Fe}$ & 0,02 & $-0,47$ \\
$\mathrm{Mn}$ & $-0,03$ & $-0,29$ \\
$\mathrm{Zn}$ & 0,33 & 0,05 \\
$\mathrm{DBO} / \mathrm{DQO}$ & 0,34 & 0,04 \\
\hline
\end{tabular}

celda, por esta razón aparecen con valores de carga altos las variables DBO, DQO y STV. El segundo componente principal CP2, da explicación a lo relacionado a la actividad biológica (relacionadas con el control de acidez del medio), apareciendo con mayor carga en este componente las variables $\mathrm{NT}, \mathrm{N}_{-} \mathrm{NH}_{3}$ y alcalinidad. Así se concluye que CP2 esta mas relacionado con la actividad biológica.

La Figura 1 muestra el comportamiento de las variables fisicoquímicas (scores en el espacio de los componentes principales para la celda 4), después de haber realizado pretratamiento a los datos, utilizando auto escalado, (auto scaling), en la cual se substrae la media para cada variable seguida por la división por la desviación estándar.

La importancia de cada una de de las variables que se muestran es directamente proporcional a su longitud vectorial a partir del origen del grafico, y así mismo, el ángulo entre dos variables es inversamente proporcional a la correlación entre ellas (a menor ángulo existe mayor correlación y viceversa).

En la Figura 1 se observa que el eje correspondiente al componente principal CP1, explica un $49.8 \%$ de la variabilidad total, mientas el CP2 explica el $16 \%$. Se asumieron como satisfactorios los dos primeros componentes principales al explicar un $66 \%$ de la variación muestral, lo cual equivale a pasar de 12 variables a solo 2 componentes principales. En esta figura se puede observar el comportamiento de las variables; la importancia de cada variable es proporcional a su longitud vectorial, y así mismo, el ángulo entre dos variables es inversamente proporcional a la correlación entre ellas. Por ejemplo el ángulo entre las variables DBO y DQO es pequeño, mostrando una alta correlación entre ellas, mientras que la variable Alcalinizad no esta nada correlacionada con la variable Fe. De igual manera se observa una correlación negativa entre Alcalinidad y Fe.

En la Figura 2 se observa que la medida que el tiempo pasa las variables que hacen parte del componente principal 1(CP1) en el espacio del tiempo en el caso de la celda C4 disminuyen su valor, porque a medida que pasa el tiempo o cuanto mayor es el valor en meses de la variable (edad de la celda), los valores de CP1 tienden a disminuir. 


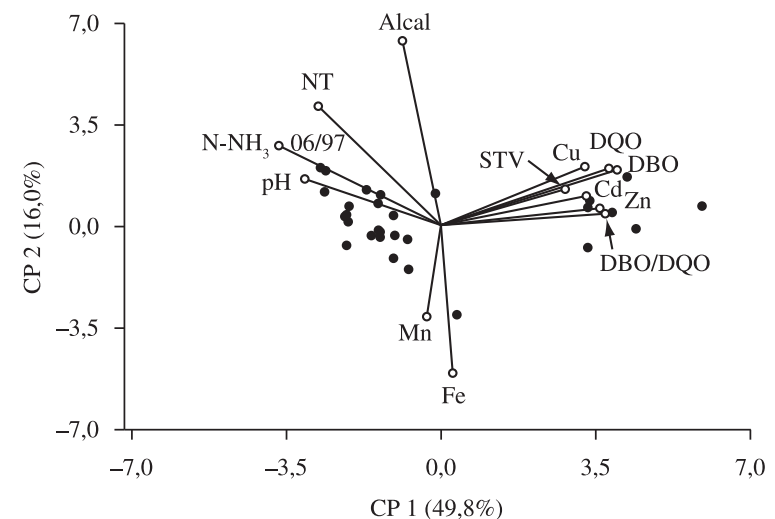

Figura 1. Grafico biplot de los scores en el espacio de los componentes. Principales para la celda 4. Pretratamiento usando auto scaling.

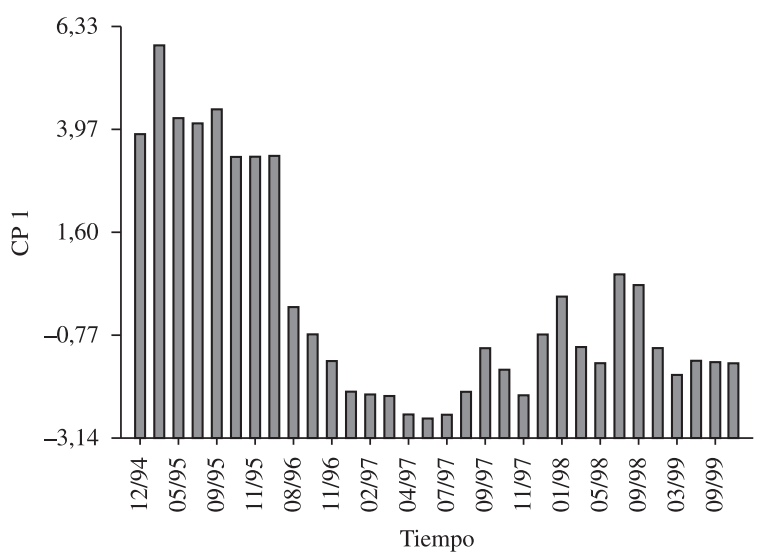

Figura 2. Scores PC1 vs tiempo para la celda 4.

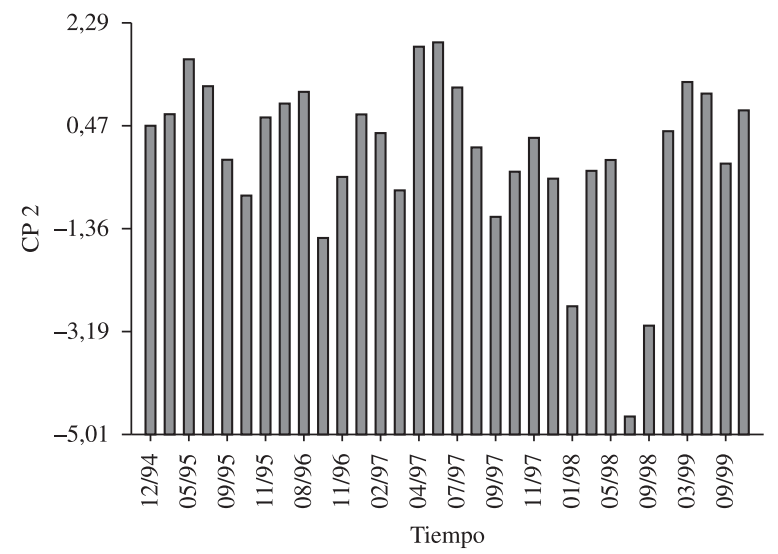

Figura 3. Scores PC2 vs tiempo para la celda 4.

A medida que pasa el tiempo de la celda, los valores de CP2 tienden a aumentar (Figura 3), pues en el periodo de degradación de materia orgánica los microorganismos involucrados en la degradación de materia liberan sustancias y ocurre el incremento de actividad biológica.

La evolucion de los scores en el tiempo en la celda 4 a 5 años la medida que el tiempo pasa las variables que hacen parte del componente principal $1(\mathrm{CP} 1)$ en el espacio del tiempo disminuyen su valor, porque a medida que pasa el tiempo o cuanto mayor es el valor en meses de la variable (edad de la celda), los valores de CP1 tienden a disminuir.
Esto explica que a medida que pasa el tiempo, mayor es la edad de la celda, la actividad de degradación disminuye y la actividad biológica se incrementa.

Los resultados del análisis PCA, describen la estructura y las interrelaciones de variables originales en el fenómeno de biodegradación de materia orgánica a partir del análisis e interpretación de los componentes principales, obtenidos agrupando variables que ofrecen informaciones semejantes, que están altamente correlacionadas.

El lixiviado es el resultado de la acción enzimática de los microorganismos en los productos resultantes de la biodegradación. En la degradación anaeróbica el nitrógeno es importante para la determinación de la suficiencia nutritiva de las condiciones de cada fase de estabilización.

Los parámetros importantes para degradación de materia orgánica son: pH, ST, Alcalinidad, DQO, DBO, Nitrógeno total y Nitrógeno amoniacal. Los parámetro que miden la biodegradabilidad son : DQO y DBO.

Un indicativo de actividad biológica llevada a cabo en la celda 4 del relleno sanitario se observa cuando la concentración de nitrógeno amoniacal es superior a la concentración de nitrógeno orgánico.

Con respecto a la relación $\mathrm{DBO} / \mathrm{DQO}$, la cual reporta la biodegradabilidad, se observa que hasta los primeros 5 años se tienen muchos microorganismos para degradación, teniéndose valores mayores para esta relación (0.34). Se puede concluir también, en la Figura 1, que pasados 5 años continua ocurriendo actividad biológica, pues se observa una intensidad del nitrógeno total muy alta.

\section{Agradecimientos}

Los autores agradecen a la Universidade de Caxias do Sul, Brazil, il Programa de Automatización, Universidad de Ibagué-Colombia e Facultad Ingeniería Agronómica, Universidad del Tolima Colombia y sus programas de cooperación.

\section{Refencias Bibliograficas}

1. Gu, J. - Int. Biodeterior. Biodegrad., 52, p.69-91 (2003).

2.Zaterra, A.; Bianchi, O.; Peresin, D.; Schneider, V. E.; Brandalise, R. N. \& Zeni, M. - "Índices de Geração de Resíduos Poliméricos no Município de Caxias do Sul através da Caracterização Física e Composição Gravimétrica", in Anais do $7^{\circ}$ Congresso Brasileiro de Polímeros, Belo Horizonte - MG, set. (2003).

3. American Public Health Association - APHA; American Water Works Association - AWWA \& Water Pollution Control Federation - WPCF. "Standard methods for examination of water and waste-water", 17. ed., New York (1989).

4. Albertsson, A. \& Karlsson, S. - Int. Biodeterior. Biodegrad., 31, p.161-170 (1993). 
5. Vazzoler, R. F. - "Microbiologia e Saneamento Ambiental”, USP, São Paulo (2001).

6. Robinson, H. D. \& Maris, P. J. - "Leachate from domestic waste: generation, composition and Treatment: a review”, VRC Technical Report TR 108 (1979).

7. Grisa, A. M. C. \& Zeni, M. - "Análise termogravimétrica commoditie polímero polipropileno (PP) em aterro sanitário - II", in: Anais do $8^{\circ}$ Congresso Brasileiro de Polímeros, Águas de Lindóia, São Paulo, nov. (2005).

8. Rabello, M. - "Aditivação de polímeros", Artliber, São Paulo (2000).

9. Pérez, C. - "Técnicas Estatísticas com SPSS", Prentice Hall, Madrid (2001).

10. Lema, T. A. - "Borrador para algunos elementos de statistical multivariada", Dissertação de Mestrado, Universidad Nacional de Colombia, Medellín, Colômbia (1996).

11. Smith, L. I. - "A tutorial on principal components analysis", University of Otago, Dunedin, 2002.

12. Poppi, R. J.; Sena, M. M. de -“ Avaliação do uso de métodos quimiométricos em análise de solo", Quím. Nova, 23, 4 (2000).

13. Oliveira, F. R. P; Goldberg, K.; Liese, A. \& Hitzmann, B. - Chemometrics and Intelligent Laboratory systems, 94, p.118-122 (2008).

14. Sol- Larranaga, C.; Navarro-Biasco, I. - Food Chemistry, 112, p.189-196 (2009).

15. Fermo, P.; Delnevo, E.; Lasagni, M.; Polla, S. \& Vos, P. Microchemical Journal, 88, p.150-159 (2008).

16. Malinowski, E. R. - "Factor Analysis in Chemistry", 2 ed., John Wiley, New York (1991).
17. Grisa, A. - "Estudio de la degradación de los polímeros commodities en el medio ambiente", Tese de Doutorado, Universidad de Leon, Espanha (2004).

18. Marcilla, A.; Gomez, A.; Reyes-Labarta, J. A. \& Giner, A. - Polym. Degrad. Stab., 80, p.233-240 (2003).

19. Bonelli, C. M. C.; Martins, A. F.; Mano, E. B. \& Beatty, C. L. - Journal of Applied Polymer Science, 80, p.1305-1311 (2001).

20. Billingham, N.C. \& Calved, P.D. "An Introduction Degradation and Stabilization of Polyolefins", Aplied Science Publishers, London (1983).

21. Hinsken, H.; Moss, S.; Pauquet, J. R. \& Zweifel, H.Polym. Degrad. Stab., 34, p.279-293 (1991).

22. Kelen, T. "Polymer Degradation", Van nostrad Reinhold, New York (1983).

23. Suat, U.; Ahmet, R.; Ozkan, J. Y. \& Selhan, K. - Fuel Process Technol., 89, 11, p.1148-1152 (2008).

24. Chan, J. H. Balkes. T. - Polym. Degrad. and Stab., 57, p.135-149, (1997).

25. Severine, F.; Gallo R. \& Ipsale S. - Polym. Degrad. and Stab., 22, p.185-194, (1998).

26. Grisa, A.M.C. and Zeni, M.- Revista Iberoamericana de Polímeros, 9, p.19-24 (2008).

27. Catalina, F. - Revista de Plásticos Modernos, 75, p.502 (1998).

Enviado: 11/06/09

Reenviado: 04/02/10

Aceito: $11 / 02 / 10$

DOI: $10.1590 /$ S0104-14282010005000020 